\title{
On the Uniqueness and Asymptotic Behaviour of Plasma Corners
}

\author{
M. Kunze
}

\begin{abstract}
In [2] the existence of solutions to a certain free boundary problem describing a plasma-vacuum interface in plane geometry was shown. This paper is a supplement to the investigation of $R$. Kaiser and D. Lortz in [2]: We shall first remark that the existence of such solutions (so-called plasma corners) can be proved by using a more general approach. Then we shall show uniqueness for small parameters, and finally we shall derive exact formulas describing the asymptotic behaviour of solutions at the corner.
\end{abstract}

Keywords: Free boundary problems, nonlinear integral equations

AMS subject classiflcation: 76 W05

\section{Introduction}

The purpose of this paper is to investigate properties of solutions to the nonlinear integral equation.

$$
x^{2}(t)-t^{2}+\frac{J}{4 \pi} \int_{0}^{1} k(t ; s, x(t), x(s)) d s=0 \quad(t \in[0,1])
$$

where $J \geq 0$ is a parameter, and the kernel $k$ is defined as

$$
\begin{aligned}
k(t, s, y, x)=x & \ln \left(\frac{\left.\left((x-y)^{2}+(s-t)^{2}\right)(x+y)^{2}+(s-t)^{2}\right)}{\left(x^{2}+s^{2}\right)^{2}}\right) \\
& +y \ln \left(\frac{(x+y)^{2}+(s-t)^{2}}{(x-y)^{2}+(s-t)^{2}}\right) \\
& +2(s-t)\left(\arctan \frac{x-y}{s-t}+\arctan \frac{x+y}{s-t}-2 \arctan \frac{x}{s}\right) .
\end{aligned}
$$

Note that equation (1) is a non-standard nonlinear integral equation, because the kernel $k$ is $y=x(t)$-dependent in a non-trivial way.

We shall start by giving a short outline of the physics which motivate the study of equation (1). Following [6] or [2] (see also [1] and [8] for additional remarks), consider

M. Kunze: Ludwig-Maximilian-Univ., Math. Inst., Theresienstr. 39, D - 80333 München. e-mail: kunze@rz.mathematik.uni-muenchen.de 
a two-dimensional plasma in plane $(t, y)$-geometry. The plasma is supposed to fill the domain $D_{p} \subset \mathbb{R}^{2}$ and to satisfy

$$
\begin{aligned}
\Delta \psi+J & =0 & & \text { in } D_{p} \\
\Delta \hat{\psi} & =0 & & \text { in } D_{\nu} \\
\psi=\hat{\psi} & =0 & & \text { on } \partial D_{p} \\
\frac{\partial \psi}{\partial n} & =\frac{\partial \hat{\psi}}{\partial n} & & \text { on } \partial D_{p} \\
\hat{\psi} & \rightarrow \hat{\psi}_{\text {ext }} & & \text { as }|(t, y)| \rightarrow \infty .
\end{aligned}
$$

Here $D_{v}=\mathbb{R}^{2} \backslash D_{p}$ denotes the domain filled by vacuum, $\psi=\psi(t, y)$ and $\bar{\psi}=\hat{\psi}(t, y)$ are the flux functions in $D_{p}$ and $D_{v}$, respectively, and $\hat{\psi}_{\text {ext }}$ is a prescribed external field. Furthermore, the parameter $J \geq 0$ denotes the constant (torodial) current density, and $\frac{\partial}{\partial n}$ indicates differentiation in the direction of the exterior normal $n$ to the boundary $\partial D_{p}$ of the domain $D_{p}$. Now solving the free boundary problem (3) is tantamount to finding the functions $\psi$ and $\tilde{\psi}$, the parameter $J \geq 0$, and the boundary $\partial D_{p}=\partial D_{v}$ such that equations (3) are satisfied.

For simplification it is reasonable to choose $\hat{\psi}_{\text {ext }}$ of the form

$$
\hat{\psi}_{\text {ext }}(t, y)=\psi_{0}+\psi_{1} t+t^{2}-y^{2}
$$

with $\psi_{0}, \psi_{1} \in \mathbb{R}$. We also assume that $D_{p}$ is a symmetric set which satisfies (in suitably chosen coordinates) $(0,0) \in \partial D_{p}, D_{p} \subset[0, \infty) \times \mathbb{R}$, and $(t, y) \in D_{p}$ if and only if $(t,-y) \in D_{p}$ for $(t, y) \in[0, \infty) \times \mathbb{R}$. Furthermore, we suppose that the upper part of the boundary $\partial D_{p}$ can be described by a continuous function $x:[0, \infty) \rightarrow[0, \infty)$. Then $\partial D_{p} \cap([0, \infty) \times(-\infty, 0])$ is given by the function $-x$. Because we are mainly interested in understanding the local behaviour of the plasma in a neighbourhood of the origin, we restrict ourselves to considering $\left.x\right|_{[0,1]}$. Therefore we have to impose the additional boundary conditions

$$
\begin{aligned}
\psi & =\hat{\psi} & & \text { on }\left(\partial D_{p}^{a}\right)_{1} \\
\frac{\partial \psi}{\partial n} & =\frac{\partial \hat{\psi}}{\partial n} & & \text { on }\left(\partial D_{p}^{a}\right)_{1}
\end{aligned}
$$

where we define $D_{p}^{a}=\left\{(t, y) \in D_{p}: t \leq 1\right\}$ and $\left(\partial D_{p}^{a}\right)_{1}=D_{p} \cap(\{1\} \times \mathbb{R})$. The following picture which is taken from [2: p. 166] may serve as an illustration.

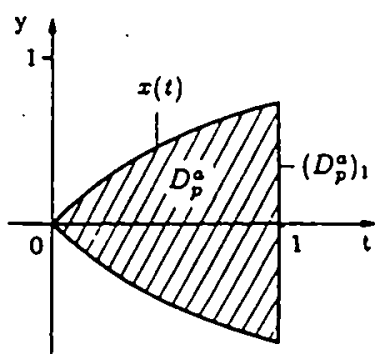

Fig. 1. Qualitative sketch of the domain $D_{p}^{a}$ 
If we think, e.g., of cleaning the plasma, it sometimes can be useful to have a stagnation point (a so-called plasma corner) at the origin. Then

$$
\nabla \psi(0,0)=0
$$

has to be satisfied. To solve (3), (5), and (6), we make the ansatz

$$
\left.\begin{array}{l}
\psi(t, y) \\
\hat{\psi}(t, y)
\end{array}\right\}=\hat{\psi}_{\text {ext }}(t, y)+J \int_{D_{p}^{a}} G(t, s, y, x) d s d x
$$

Here

$$
G(t, s, y, x)=-\frac{1}{4 \pi} \ln \left((x-y)^{2}+(s-t)^{2}\right)
$$

denotes the Green function for the Laplacian in $\mathbb{R}^{2}$ (see [2] from equation (2.10)). Since

$$
\int_{D_{p}^{a}} G(t, s, y, x) d s d x=\int_{0}^{1} d s \int_{-x(s)}^{x(s)} d x G(t, s, y, x)
$$

we obtain a formula for $\psi$ which allows us to express $\psi_{0}$ and $\psi_{1}$ in (4) by means of $\psi(0,0)=0$ and (6). Using $\psi(t, x(t))=\hat{\psi}(t, x(t))=0$ for $t \in[0,1]$, we finally arrive at the nonlinear integral equation (1) for the continuous function $x:[0,1] \rightarrow[0, \infty)$ describing the upper part of the plasma's boundary cut off at $t=1$ (see Figure 1). Therefore the free boundary problem was reduced to determining $x$.

Now we turn to the description of the contents of this paper. When investigating (classical) free boundary problems, results on the uniqueness of solutions in the case of a small parameter were obtained, too (see [7], [9], and many other papers). Since $x(t)=t(t \in[0,1])$ is the only continuous non-negative solution of equation (1) for $J=0$, and since the solutions of equation (1) are to be interpreted as describing the free boundary, it is therefore natural to ask whether or not such results on the uniqueness of solutions also hold for equation (1). In Section 2 we will show that this question may be answered positively in that there is one and only one continuous non-negative solution of equation (1) for parameters $J>0$ sufficiently small. Next, in Section 3 we shall derive exact formulas describing the asymptotic behaviour of solutions $x=x(t)$ of equation (1) as $t \rightarrow 0^{+}$. In particular, these formulas will prove that these solutions form an angle of $\pi / 4$ with the $t$-axis. Therefore one can speak of a plasma corner.

To avoid technicalities, we added an Appendix where all properties of the kernel $\boldsymbol{k}$ are collected which were used in Sections 2 and 3.

Before turning to the main topics of this paper, let us note that a proof for the existence of solutions to equation (1) being more elegant than the one given in [2] can be obtained by using a general theorem on the existence of zeros of so-called semicondensing maps (cf. [5]). Generally speaking, this theorem may be applied when dealing with nonlinear integral equations of the type

$$
\varphi(t, x(t))+\int_{0}^{1} \psi(t, s, x(t), x(s)) d s=0 \quad(t \in[0,1])
$$

in case that the equation shows a certain monotonicity in $y=x(t)$ (note that $\partial_{y} k \geq 0$ in (1), cf. Lemma A.1 in the Appendix) and a certain compactness in $x=x(s)$. 


\section{On the uniqueness of solutions}

Let us define the sets of continuous functions

$$
C^{+}([0,1])=\{x \in C([0,1]) \mid x(t) \geq 0 \text { for all } t \in[0,1]\}
$$

and, for $\zeta \in[0,1]$,

$$
C_{\zeta}([0,1])=\{x \in C([0,1]) \mid \zeta t \leq x(t) \leq t \text { for all } t \in[0,1]\}
$$

Then, by [2], there is a number $J_{1}>0$ such that equation (1) has a solution $x \in$ $C^{+}([0,1])$ whenever $J \in\left[0, J_{1}\right]$. We are going to show the following

Theorem 2.1: There is a number $J_{*}>0$ such that for all parameters $J \in\left[0, J_{*}\right)$ there exists a unique solution of equation $(1)$ in $C^{+}([0,1])$.

The proof of that theorem will be divided into several lemmas.

Lemma 2.2: Let $x \in C^{+}([0,1])$ be a solution of equation (1) for some parameter $J \geq 0$. Then the following statements are true:

a) $x \in C_{0}([0,1])$.

$$
\begin{aligned}
& \text { b) }\left(t^{2}-x^{2}(t)\right)\left(1+\frac{J}{2 \pi} \cdot \int_{t}^{1} \frac{\tilde{x}(s)}{1+\tilde{x}^{2}(s)} \frac{d s}{s}\right) \\
& =\frac{J}{8 \pi} t^{2}\left(2 \int_{1}^{\infty} \frac{d \xi}{\xi^{3}} I\left(\tilde{x}\left(\frac{t}{\xi}\right), \tilde{x}(t), \xi\right)+\int_{t}^{1} d \xi \int_{0}^{1} d s(1-s)^{2} \partial_{\xi \xi \xi}^{3} I\left(\tilde{x}\left(\frac{t}{\xi}\right), \tilde{x}(t), s \xi\right)\right)
\end{aligned}
$$

for $t \in(0,1]$, where $\tilde{x}(\tau)=\frac{x(r)}{\tau}$ for $\tau \in(0,1]$.

c) $x \in C_{\zeta}([0,1])$ if $J \leq J_{0}$, where $J_{0}=J_{0}(\zeta)>0$ is a number existing for every $\zeta \in(0,1)$.

Proof: a) For fixed $t$ the function

$$
y \longrightarrow \Psi^{x}(t, y)=y^{2}-t^{2}+\frac{J}{4 \pi} \int_{0}^{1} k(t, s, y, x(s)) d s \quad(y \in[0, \infty))
$$

is differentiable with $\partial_{y} \Psi^{x}(t, y) \geq 2 y$ (see Lemma A.1 of the Appendix). Furthermore, Lemma A.3/c) of the Appendix and the fact that $x$ is a solution of equation (1) yield $\Psi^{x}(t, t) \geq 0$ as well as $\Psi^{x}(t, x(t))=0$ on $[0,1]$, and therefore the claim.

b) Using statement a) we see that $x \in C_{0}([0,1])$, hence $0 \leq \tilde{x}(\tau) \leq 1$ for $\tau \in(0,1]$. Let $t \in(0,1]$ be fixed. Lemma A.3/i) of the Appendix shows that both integrals on 
the right-hand side of the claimed equality do exist. By means of Lemma A.3/b) and Lemma A.2/e) of the Appendix we obtain

$$
\begin{aligned}
0= & x^{2}(t)-t^{2}+\frac{J}{4 \pi} \int_{0}^{1} k(t, s, x(t), x(s)) d s \\
= & x^{2}(t)-t^{2}+\frac{J}{4 \pi} t^{2}\left(\int_{1}^{\infty} \frac{d \xi}{\xi^{3}} I\left(\tilde{x}\left(\frac{t}{\xi}\right), \tilde{x}(t), \xi\right)+\int_{t}^{1} \frac{d \xi}{\xi^{3}} I\left(\tilde{x}\left(\frac{t}{\xi}\right), \tilde{x}(t), \xi\right)\right) \\
= & x^{2}(t)-t^{2}+\frac{J}{4 \pi} t^{2} \int_{1}^{\infty} \frac{d \xi}{\xi^{3}} I(\ldots) \\
& +\frac{J}{4 \pi} t^{2} \int_{t}^{1} \frac{d \xi}{\xi^{3}}\left(-2\left(1-\tilde{x}^{2}(t)\right)\left(\frac{\tilde{x}(t / \xi)}{1+\tilde{x}^{2}(t / \xi)}\right) \xi^{2}\right. \\
& \left.+\frac{1}{2} \xi^{3} \int_{0}^{1} d s(1-s)^{2} \partial_{\xi \xi \xi}^{3} I\left(\tilde{x}\left(\frac{t}{\xi}\right), \tilde{x}(t), s \xi\right)\right) \\
= & \left(x^{2}(t)-t^{2}\right)\left(1+\frac{J}{2 \pi} \int_{t}^{1} \frac{d \xi}{\xi} \frac{\tilde{x}(t / \xi)}{1+\tilde{x}^{2}(t / \xi)}\right)+\frac{J}{4 \pi} t^{2} \int_{1}^{\infty} \frac{d \xi}{\xi^{3}} I(\ldots) \\
& +\frac{J}{8 \pi} t^{2} \int_{t}^{1} d \xi \int_{0}^{1} d s(1-s)^{2} \partial_{\xi \xi \xi}^{3} I\left(\tilde{x}\left(\frac{t}{\xi}\right), \tilde{x}(t), s \xi\right) .
\end{aligned}
$$

Using the transformation $s=\frac{t}{\xi}$ and $\frac{d \xi}{\xi}=-\frac{d s}{s}$ in the first integral completes the proof of statement $\mathbf{b})$.

c) Fix $\zeta \in(0,1)$ and let $C_{1}$ denote the constant from Lemma A.3/i) of the Appendix. We define $J_{0}(\zeta)=\frac{1-\zeta^{2}}{2 C_{1}}$. If $x \in C^{+}([0,1])$ is a solution of equation (1) corresponding to the parameter $J \leq J_{0}$, then part a) implies $x(0)=0$ and $\tilde{x}(\tau) \in[0,1]$ on $(0,1]$. Hence statement b) and Lemma A.3/i) of the Appendix yield

$$
\begin{aligned}
0 \leq t^{2}-x^{2}(t) \leq & \left(t^{2}-x^{2}(t)\right)\left(1+\frac{J}{2 \pi} \int_{t}^{1} \frac{\tilde{x}(s)}{1+\tilde{x}^{2}(s)} \frac{d s}{s}\right) \\
= & \frac{J}{4 \pi} t^{2} \int_{1}^{\infty} \frac{d \xi}{\xi^{3}} I\left(\tilde{x}\left(\frac{t}{\xi}\right), \tilde{x}(t), \xi\right) \\
& +\frac{J}{8 \pi} t^{2} \int_{t}^{1} d \xi \int_{0}^{1} d s(1-s)^{2} \partial_{\xi \xi \xi}^{3} I\left(\tilde{x}\left(\frac{t}{\xi}\right), \tilde{x}(t), s \xi\right) \\
\leq & 2 C_{1} J t^{2} .
\end{aligned}
$$

for $t \in(0,1]$. Consequently, $x^{2}(t) \geq\left(1-2 C_{1} J\right) t^{2} \geq \zeta^{2} t^{2}$, and therefore $x \in C_{\zeta}([0,1])$ 
The next preparatory lemma already contains some information about the asymptotic behaviour of solutions for $t \rightarrow 0^{+}$.

Lemma 2.3: There is a constant $C>0$ such that

$$
\left(t^{2}-x^{2}(t)\right)(1-\ln t) \leq C \frac{t^{2}}{\zeta} \quad(t \in(0,1])
$$

for all $\zeta \in(0,1]$ and solutions $x \in C_{\zeta}([0,1])$ of equation (1) corresponding to some parameter $J \geq 0$.

Proof: Let again $C_{1}$ denote the constant from Lemma A.3/i) of the Appendix and let $C=1+8 \pi C_{1}$. If $x$ is a solution of equation (1) for $J=0$, then the claimed estimate is obvious. In the case $J>0$ we obtain

$$
\begin{aligned}
\left(t^{2}-x^{2}(t)\right)(-\ln t) & =\left(t^{2}-x^{2}(t)\right) \int_{t}^{1} \frac{d s}{s} \\
& \leq \frac{2}{\zeta}\left(t^{2}-x^{2}(t)\right) \int_{t}^{1} \frac{\tilde{x}(s)}{1+\tilde{x}^{2}(s)} \frac{d s}{s} \\
& \leq \frac{4 \pi}{\zeta J}\left(t^{2}-x^{2}(t)\right)\left(1+\frac{J}{2 \pi} \int_{t}^{1} \frac{\tilde{x}(s)}{1+\tilde{x}^{2}(s)} \frac{d s}{s}\right) \\
& \leq \frac{4 \pi}{\zeta J} 2 C_{1} J t^{2}=8 \pi C_{1} \frac{t^{2}}{\zeta}
\end{aligned}
$$

for $t \in(0,1]$, by the estimate (7). Furthermore, $\left(t^{2}-x^{2}(t)\right) \leq\left(1-\zeta^{2}\right) t^{2} \leq \frac{t^{2}}{\zeta}$ because of $x(t) \geq \zeta$ on $[0,1]$. Adding these two estimates completes the proof of the lemma

To show uniqueness, we need to compare solutions of equation (1). This will be done in the next

Lemma 2.4: There is a constant $C>0$. such that for all solutions $x, y \in C_{\frac{1}{2}}([0,1])$ of equation (1) corresponding to the same parameter $J \geq 0$

$$
\begin{aligned}
\Delta_{0}(t) \leq C J & \left(\Delta_{0}(t)+\frac{1}{1-\ln t} \int_{t}^{1} \Delta_{0}(s) \frac{d s}{s}\right. \\
& \left.+\frac{1}{t^{2}} \int_{0}^{t} \Delta_{0}(s) h\left(\frac{t}{s}\right) s d s-\int_{i}^{1} \frac{d s}{s} \ln (1-s) \Delta_{0}\left(\frac{t}{s}\right)\right)
\end{aligned}
$$

holds on $(0,1]$. Here we let $\Delta_{0}(t)=\left|\tilde{x}^{2}(t)-\tilde{y}^{2}(t)\right|$ for $t \in(0,1]$ and $h(\xi)=\xi+\left|\ln (1-\xi)^{2}\right|$ for $\xi \in[0, \infty) \backslash\{1\}$.

Proof: Let $x, y \in C_{\frac{1}{2}}([0,1])$ be as in the lemma. Note that

$$
\Delta_{0}(\tau) \geq|\tilde{x}(\tau)-\tilde{y}(\tau)|
$$


because of $\tilde{x}(\tau), \tilde{y}(\tau) \geq \frac{1}{2}$ on $(0,1]$. Furthermore, for $s \in(0,1]$ we obtain the estimate

$$
\begin{aligned}
\left|\frac{\tilde{x}(s)}{1+\tilde{x}^{2}(s)}-\frac{\tilde{y}(s)}{1+\tilde{y}^{2}(s)}\right| & =\frac{\left|\tilde{x}(s)\left(1+\tilde{y}^{2}(s)\right)-\tilde{y}(s)\left(1+\tilde{x}^{2}(s)\right)\right|}{\left(1+\tilde{x}^{2}(s)\right)\left(1+\tilde{y}^{2}(s)\right)} \\
& \leq(1-\tilde{x}(s) \tilde{y}(s))|\tilde{x}(s)-\tilde{y}(s)| \\
& \leq|\tilde{x}(s)-\tilde{y}(s)| \\
& \leq \Delta_{0}(s)
\end{aligned}
$$

and Lemma 2.3 implies the existence of a constant $C>0$, independent of $x, y$ and $J$, such that

$$
\left(1-\tilde{x}^{2}(t)\right) \leq \frac{C}{1-\ln t} \quad(t \in(0,1])
$$

For $\xi>1$ we have $I(\cdot, \cdot, \xi) \in C^{1}\left([0,1]^{2}\right)$ by Lemma A.2 of the Appendix. Hence, applying Lemma A.3/d) and e) of the Appendix it follows that, for all $t \in(0,1]$ and $\xi>1$,

$$
\begin{aligned}
& \left|I\left(\tilde{x}\left(\frac{t}{\xi}\right), \tilde{x}(t), \xi\right)-I\left(\tilde{y}\left(\frac{t}{\xi}\right), \tilde{y}(t), \xi\right)\right| \\
& \quad \leq C\left(\left|\tilde{x}\left(\frac{t}{\xi}\right)-\tilde{y}\left(\frac{t}{\xi}\right)\right| h_{2}(\xi)+|\tilde{x}(t)-\tilde{y}(t)| h_{3}(\xi)\right) \\
& \quad \leq C\left(\Delta_{0}\left(\frac{t}{\xi}\right) h_{2}(\xi)+\Delta_{0}(t) h_{3}(\xi)\right)
\end{aligned}
$$

holds with a constant $C>0$ independent of $x, y$, and $J$, and $h_{2}(\theta)=\theta+\left|\ln (1-\theta)^{2}\right|$ on $[0, \infty) \backslash\{1\}$, hence $\left(\theta \rightarrow \frac{h_{2}(\theta)}{\theta^{3}}\right) \in L_{1}([1, \infty))$. Analogously, for $t \in(0,1], s \in[0,1]$, and $\xi \in[0,1)$ we have

$$
\begin{aligned}
& \left|\partial_{\xi \xi \xi}^{3} I\left(\tilde{x}\left(\frac{t}{\xi}\right), \tilde{x}(t), s \xi\right)-\partial_{\xi \xi \xi}^{3} I\left(\tilde{y}\left(\frac{t}{\xi}\right), \tilde{y}(t), s \xi\right)\right| \\
& \quad \leq C\left(\left|\tilde{x}\left(\frac{t}{\xi}\right)-\tilde{y}\left(\frac{t}{\xi}\right)\right|+|\tilde{x}(t)-\tilde{y}(t)|\right) \frac{1}{(1-s \xi)^{3}} \\
& \quad \leq C\left(\Delta_{0}\left(\frac{t}{\xi}\right)+\Delta_{0}(t)\right) \frac{1}{(1-s \xi)^{3}}
\end{aligned}
$$

by Lemma A.3/g) and h) (cf. Lemma A.2/d)) of the Appendix, for some constant $C>0$, again independent of $x, y$, and $J$. By means of Lemma 2.2/b), (10), (9), (11), 
636

M. Kunze

and (12) we obtain

$$
\begin{aligned}
\Delta_{0}(t) \leq & \left|\left(\left(1-\tilde{x}^{2}(t)\right)-\left(1-\tilde{y}^{2}(t)\right)\right)\left(1+\frac{J}{2 \pi} \int_{t}^{1} \frac{\tilde{y}(s)}{1+\tilde{y}^{2}(s)} \frac{d s}{s}\right)\right| \\
\leq & \frac{J}{2 \pi}\left(1-\tilde{x}^{2}(t)\right) \int_{t}^{1}\left|\frac{\tilde{x}(s)}{1+\tilde{x}^{2}(s)}-\frac{\tilde{y}(s)}{1+\tilde{y}^{2}(s)}\right| \frac{d s}{s} \\
& +\mid\left(1-\tilde{x}^{2}(t)\right)\left(1+\frac{J}{2 \pi} \int_{t}^{1} \frac{\tilde{x}(s)}{1+\tilde{x}^{2}(s)} \frac{d s}{s}\right) \\
& -\left(1-\tilde{y}^{2}(t)\right)\left(1+\frac{J}{2 \pi} \int_{t}^{1} \frac{\tilde{y}(s)}{1+\tilde{y}^{2}(s)} \frac{d s}{s}\right) \mid \\
\leq & C J\left(\frac{1}{1-\ln t} \int_{t}^{1} \Delta_{0}(s) \frac{d s}{s}\right. \\
& +\int_{1}^{\infty} \frac{d \xi}{\xi^{3}}\left|I\left(\tilde{x}\left(\frac{t}{\xi}\right), \tilde{x}(t), \xi\right)-I\left(\tilde{y}\left(\frac{t}{\xi}\right), \tilde{y}(t), \xi\right)\right| \\
& \left.+\int_{t}^{1} \frac{d \xi}{d} \int_{0}^{1} d s(1-s)^{2}\left|\partial_{\xi \xi \xi}^{3} I\left(\tilde{x}\left(\frac{t}{\xi}\right), \tilde{x}(t), s \xi\right)-\partial_{\xi \xi \xi}^{3} I\left(\tilde{y}\left(\frac{t}{\xi}\right), \tilde{y}(t), s \xi\right)\right|\right) \\
\leq & C J\left(\frac{1}{1-\ln t} \int_{t}^{1} \Delta_{0}(s) \frac{d s}{s}\right. \\
& +\int_{1}^{\infty} \frac{d \xi}{\xi^{3}}\left(\Delta_{0}\left(\frac{t}{\xi}\right) h_{2}(\xi)+\Delta_{0}(t) h_{3}(\xi)\right)
\end{aligned}
$$

whenever $t \in(0,1]$. Now

$$
\left|\frac{h_{3}}{i d^{3}}\right|_{L_{1}([1, \infty))}=C<\infty
$$

and

$$
\quad \quad \quad \int_{t}^{1} d \xi \int_{0}^{1} d s \frac{(1-s)^{2}}{(1-s \xi)^{3}} \leq \frac{\pi^{2}}{6} \quad(t \in[0,1])
$$


hence

$$
\begin{aligned}
\Delta_{0}(t) \leq & C J\left(\Delta_{0}(t)+\frac{1}{1-\ln t} \int_{t}^{1} \Delta_{0}(s) \frac{d s}{s}\right. \\
& \left.+\int_{1}^{\infty} \frac{d \xi}{\xi^{3}} \Delta_{0}\left(\frac{t}{\xi}\right) h_{2}(\xi)+\int_{t}^{1} d \xi \int_{0}^{1} d s \frac{(1-s)^{2}}{(1-s \xi)^{3}} \Delta_{0}\left(\frac{t}{\xi}\right)\right)
\end{aligned}
$$

for all $t \in(0,1]$ with a constant $C>0$ independent of $x, y$, and $J$. Substituting $s=\frac{t}{\xi}$ and $\frac{d \xi}{\xi^{3}}=-\frac{s d s}{t^{2}}$ yields

$$
\int_{1}^{\infty} \frac{d \xi}{\xi^{3}} \Delta_{0}\left(\frac{t}{\xi}\right) h_{2}(\xi)=\frac{1}{t^{2}} \int_{0}^{t} \Delta_{0}(s) h_{2}\left(\frac{t}{s}\right) s d s
$$

and we have

$$
\begin{aligned}
\int_{t}^{1} d \xi \int_{0}^{1} d s \frac{(1-s)^{2}}{(1-s \xi)^{3}} \Delta_{0}\left(\frac{t}{\xi}\right) & \leq \int_{t}^{1} d \xi \int_{0}^{1} d s \frac{1}{1-s \xi} \Delta_{0}\left(\frac{t}{\xi}\right) \\
& =-\int_{t}^{1} \frac{d \xi}{\xi} \ln (1-\xi) \Delta_{0}\left(\frac{t}{\xi}\right)
\end{aligned}
$$

The claim follows from (13), (14), and (15)

Now we are ready to carry out the

Proof of Theorem 2.1: Let $J_{0}\left(\frac{1}{2}\right)$ be chosen in accordance with Lemma $\left.2.2 / \dot{c}\right)$, let $C$ denote the constant from Lemma 2.4 , recall that $J_{1}>0$ was a constant such that equation (1) has a solution in $C^{+}([0,1])$ for all parameters $J \in\left[0, J_{1}\right]$, and let $\gamma=1+\left|\frac{h}{i d^{s}}\right|_{L_{1}([1, \infty))}+\pi^{2} / 6 \geq 1$ with the function $h$ from Lemma 2.4. We define $J_{*}=\min \left\{J_{1}, J_{0}\left(\frac{1}{2}\right),(2 C \gamma)^{-1}\right\}$. Now let the parameter $J \in\left[0, J_{*}\right)$ be fixed and $\theta=$ $2 C \gamma J<1$. Note that (because of $J<J_{*} \leq J_{1}$ ) we already know that a solution $x \in C^{+}([0,1])$ does exist, and we only have to prove its uniqueness. For let $y \in C^{+}([0,1])$ be another solution of equation (1) corresponding to the parameter $J<J_{*} \leq J_{0}\left(\frac{1}{2}\right)$. Lemma $2.2 / \mathrm{c})$ yields $x, y \in C_{\frac{1}{2}}([0,1])$. Retaining the notations of Lemma 2.4, 'we obtain

$$
\begin{aligned}
\Delta_{0}(t)=2 \frac{1}{2} \Delta_{0}(t) \leq & 2(1-C J) \Delta_{0}(t) \\
\leq & 2 C J\left(\frac{1}{1-\ln t} \int_{t}^{1} \Delta_{0}(s) \frac{d s}{s}\right. \\
& \left.+\frac{1}{t^{2}} \int_{0}^{t} \Delta_{0}(s) h\left(\frac{t}{s}\right) s d s-\int_{t}^{1} \frac{d s}{s} \ln (1-s) \Delta_{0}\left(\frac{t}{s}\right)\right)
\end{aligned}
$$


for $t \in(0,1]$ because of $C J<\frac{C}{2 C \gamma} \leq \frac{1}{2}$. We define

$$
M=\{\Delta \in C([0,1]): 0 \leq \Delta(t) \leq 1 \text { for all } t \in[0,1]\}
$$

and $T: M \rightarrow C([0,1])$ by

$$
(T \Delta)(t)=\left\{\begin{array}{lc}
2 C J\left(\frac{1}{1-\ln t} \int_{t}^{1} \Delta(s) \frac{d s}{s}\right. & \text { for } t \in(0,1] \\
\left.\quad+\frac{1}{t^{2}} \int_{0}^{t} \Delta(s) h\left(\frac{t}{s}\right) s d s-\int_{t}^{1} \frac{d s}{s} \ln (1-s) \Delta\left(\frac{t}{s}\right)\right) & \\
2 C \gamma J \Delta(0) & \text { for } t=0 .
\end{array}\right.
$$

Then $M \subset C([0,1])$ is closed and therefore complete. Furthermore, Lemma A.4/a), c) and $\mathrm{e})$ of the Appendix tell us that $T$ is well-defined, in that $T \Delta \in C([0,1])$ for $\Delta \in M$. Since $h(\xi) \geq 0$ on $[0, \infty) \backslash\{1\}$ as well as $-\ln (1-s) \geq 0$ on $[0,1), T$ is positive, i.e. $(T \Delta)(t) \leq(T \bar{\Delta})(t)$ on $[0,1]$ whenever $\Delta, \bar{\Delta} \in M$ are such that $\Delta(t) \leq \bar{\Delta}(t)$ on $[0,1]$. Recalling the definition of $\gamma$, Lemma A.4/b), d) and f) of the Appendix imply

$$
|T \Delta-T \bar{\Delta}| \leq 2 C \gamma J|\Delta-\bar{\Delta}|=\theta|\Delta-\bar{\Delta}|
$$

for $\Delta, \bar{\Delta} \in M$, hence in particular $|\dot{\Delta}| \leq \theta|\Delta| \leq \theta \leq 1$ for $\Delta \in M$, and therefore $T M \subset M$. Using the Banach fixed-point theorem and (17), we obtain the unique fixed-point $\Delta_{*}=0$ of $T$ in $M$ and the convergence $T^{n} \Delta \rightarrow \Delta$, =0 as $n \rightarrow \infty$ in $C([0,1])$ for every $\Delta \in M$. Now, by Lemma $2.3, \lim _{t \rightarrow 0+} \tilde{x}^{2}(t)=\lim _{t \rightarrow 0+} \ddot{y} \tilde{y}^{2}(t)=1$. Therefore $\Delta_{0}=\left|\tilde{x}^{2}-\tilde{y}^{2}\right|:(0,1] \rightarrow[0, \infty)$ can be assumed to be continuous on $[0,1]$. Since $\tilde{x}^{2}(t), \tilde{y}^{2}(t) \in[0,1]$ on $(0,1]$, we have $\Delta_{0}(t) \in[0,1]$ on $[0,1]$, hence $\Delta_{0} \in M$. Consequently,

$$
0 \leq \Delta_{0}(t) \leq\left(T \Delta_{0}\right)(t) \leq\left(\dot{T}^{2} \Delta_{0}\right)(t) \leq \ldots \leq\left(T^{n} \Delta_{0}^{*}\right)(t) \longrightarrow \Delta_{*}(t)=0
$$

as $n \rightarrow \infty$ for $t \in(0,1]$ by (16). This implies $\Delta_{0}(t)=0$ on $[0,1]$, hence $x(t)=y(t)$ on $[0,1]$ by the estimate (8). The proof of Theorem 2.1 is therefore complete.

\section{On the asymptotic behaviour of solutions}

The following theorem investigates the asymptotic behaviour of solutions as $t \rightarrow 0^{+}$. In particular, we obtain the result that solutions of equation (1) form an angle of $\pi / 4$ with the $t$-axis.

Theorem 3.1: Let $\zeta \in(0,1)$ and.let $x \in C_{\zeta}([0,1])$ be a solution of equation (1) corresponding to some parameter $J>0$. Then the following formulas hold.

а) $\lim _{t \rightarrow 0^{+}}:\left(1-\frac{x(t)}{t}\right)\left(\int_{t}^{1} \frac{x(s)}{s^{2}+x^{2}(s)} d s\right)=\frac{3}{8} \pi$. 
b) $\frac{\pi}{4}-\Phi(r) \sim \frac{3 \pi}{8 \ln r^{-1}}$.

Here $(r, \Phi(r))$ are the polar coordinates of $x$ in $(t, x)$-geometry, i.e. $r(t, x)=\sqrt{t^{2}+x^{2}}$ as well as $\Phi(t, x)=\arctan \frac{x}{t}$, and $f(r) \sim g(r)$ if and only if $\frac{f(r)}{g(r)} \rightarrow 1$ as $r \rightarrow 0+$.

Proof: a) Let $\zeta \in(0,1)$ and a solution $x \in C_{\zeta}([0,1])$ of equation (1) for some parameter $J>0$ be given. Then

$$
\lim _{t \rightarrow 0^{+}} \frac{x(t)}{t}=\lim _{t \rightarrow 0^{+}} \tilde{x}(t)=1
$$

by Lemma 2.3. Therefore $\tilde{x}=x /$ id : $(0,1] \rightarrow[\zeta, 1]$ in fact is continuous in $t=0$ with $\tilde{x}(0)=1$. To prove statement a), it is enough to show

$$
\lim _{t \rightarrow 0^{+}}\left(\left(1-\tilde{x}^{2}(t)\right) \int_{t}^{1} \frac{\tilde{x}(s)}{1+\tilde{x}^{2}(s)} \frac{d s}{s}\right)=\frac{3}{4} \pi
$$

because of

$$
\int_{i}^{1} \frac{x(s)}{s^{2}+x^{2}(s)} d s=\int_{t}^{1} \frac{\tilde{x}(s)}{1+\tilde{x}^{2}(s)} \frac{d s}{s} \quad(t \in(0,1])
$$

For a fixed $\xi>1$ we have $I(\cdot, \cdot, \xi) \in C\left([0,1]^{2}\right)$ (see Lemma A.2 of the Appendix). This yields

$$
I\left(\tilde{x}\left(\frac{t}{\xi}\right), \tilde{x}(t), \xi\right) \rightarrow I(1,1, \xi) \quad \text { as } t \rightarrow 0+
$$

and the dominated convergence theorem together with Lemma A.3/i) and $\mathrm{j}$ ) of the Appendix implies

$$
\lim _{t \rightarrow 0^{+}} \int_{1}^{\infty} \frac{d \xi}{\xi^{3}} I\left(\tilde{x}\left(\frac{t}{\xi}\right), \tilde{x}(t), \xi\right)=\int_{1}^{\infty} \frac{d \xi}{\xi^{3}} I(1,1, \xi)=\pi+\ln 2
$$

Analogously,

$$
\begin{aligned}
\lim _{t \rightarrow 0+} & \int_{\imath}^{1} d \xi \int_{0}^{1} d s(1-s)^{2} \partial_{\xi \xi \xi}^{3} I\left(\tilde{x}\left(\frac{t}{\xi}\right), \tilde{x}(t), s \xi\right) \\
& =\int_{0}^{1} d \xi \int_{0}^{1} d s(1-s)^{2} \partial_{\xi \xi \xi}^{3} I(1,1, s \xi) \\
& =\pi-2 \ln 2
\end{aligned}
$$


by Lemma A.3/i) and k) of the Appendix. Finally, Lemma 2.2/b), (18), (21), and (22) show

$$
\begin{aligned}
\lim _{t \rightarrow 0^{+}} & \left(\left(1-\tilde{x}^{2}(t)\right) \int_{t}^{1} \frac{\tilde{x}(s)}{1+\tilde{x}^{2}(s)} \frac{d s}{s}\right) \\
& =\frac{2 \pi}{J} \lim _{t \rightarrow 0+}\left(\left(1-\tilde{x}^{2}(t)\right)\left(1+\frac{J}{2 \pi} \int_{l}^{1} \frac{\tilde{x}(s)}{1+\tilde{x}^{2}(s)} \frac{d s}{s}\right)-\left(1-\tilde{x}^{2}(t)\right)\right) \\
& =\frac{2 \pi}{J}\left(\frac{J}{4 \pi}(\pi+\ln 2)+\frac{J}{8 \pi}(\pi-2 \ln 2)\right) \\
& =\frac{3}{4} \pi
\end{aligned}
$$

and that is (19).

b) At first, using (20), Lemma A.4/a) of the Appendix and (18) we obtain

$$
\begin{aligned}
& -\frac{1}{\ln \sqrt{t^{2}+x^{2}(t)}} \int_{t}^{1} \frac{x(s)}{s^{2}+x^{2}(s)} d s \\
& \quad=\left(\frac{1}{1-\ln t} \int_{t}^{1} \frac{\tilde{x}(s)}{1+\tilde{x}^{2}(s)} \frac{d s}{s}\right)\left(1+\frac{1+\ln \sqrt{1+\tilde{x}^{2}(t)}}{-\ln t-\ln \sqrt{1+\tilde{x}^{2}(t)}}\right) \\
& \quad \rightarrow \frac{1}{2} \text { as } t \rightarrow 0+.
\end{aligned}
$$

Furthermore, from the mean-value theorem and (18) it follows that

$$
\frac{1-\tilde{x}(t)}{\arctan 1-\arctan \tilde{x}(t)} \rightarrow 2 \text { as } t \rightarrow 0+\text {. }
$$

Therefore statement a), (23), and (24) imply

$$
\begin{aligned}
\frac{\pi / 4-\Phi(r)}{(3 \pi) /\left(8 \ln r^{-1}\right)}= & \frac{(\pi / 4-\Phi(r))(-\ln r)}{(3 \pi) / 8} \\
= & \frac{(1-\bar{x}(t))\left(\int_{t}^{1} \frac{x(s)}{s^{2}+x^{2}(s)} d s\right)}{(3 \pi) / 8} \\
& \times \frac{(\arctan 1-\arctan \tilde{x}(t))\left(-\ln \sqrt{t^{2}+x^{2}(t)}\right)}{(1-\tilde{x}(t))\left(\int_{t}^{1} \frac{x(s)}{s^{2}+x^{2}(s)} d s\right)} \\
& \rightarrow 1 \text { as } t \rightarrow 0+,
\end{aligned}
$$

so the proof is complete

According to [3], such an asymptotic behaviour in polar coordinates was to be expected. Finally we want to remark that this behaviour for $t \rightarrow 0^{+}$in particular is independent of the parameter $J>0$ in equation (1), and that the plasma corner may be seen only on a logarithmic scale by Theorem $3.1 / \mathrm{b}$ ). 


\section{Appendix}

In this appendix we shall first collect some essential properties of the kernel $k$ in (2) and its transformed version $I$, see Lemma A.2. Afterwards we shall give another result of mainly technical nature which was used in the main part of this paper. For a detailed proof of all these formulas and estimates, the interested reader is referred to the Appendix of [4].

Lemma A.1: Let $D_{k}=\left\{(t, s, y, x) \in[0,1]^{2} \times[0, \infty)^{2}: s>0\right.$ and $\left.s \neq t\right\}$ and $k: D_{k} \rightarrow \mathbb{R}$ be defined as in (2). Then for $(t, s, y, x) \in D_{k}$

$$
\partial_{y} k(t, s, y, x)=\ln \left(\frac{(x+y)^{2}+(s-t)^{2}}{(x-y)^{2}+(s-t)^{2}}\right) \geq 0 .
$$

Lemma A.2: Let $D_{I}=\left\{(\eta, \vartheta, \xi) \in[0, \infty)^{3}: \xi \neq 1\right\}$ and $I: D_{I} \rightarrow R$ defined by

$$
\begin{aligned}
I(\eta, \vartheta, \xi)= & \eta \ln \left(\frac{\left((\eta+\xi \vartheta)^{2}+(1-\xi)^{2}\right)\left((\eta-\xi \vartheta)^{2}+(1-\xi)^{2}\right)}{\left(1+\eta^{2}\right)^{2}}\right) \\
& +\xi \vartheta \ln \left(\frac{(\eta+\xi \vartheta)^{2}+(1-\xi)^{2}}{(\eta-\xi \vartheta)^{2}+(1-\xi)^{2}}\right) \\
& +2(1-\xi)\left(\arctan \frac{\eta+\xi \vartheta}{1-\xi}+\arctan \frac{\eta-\xi \vartheta}{1-\xi}-2 \arctan \eta\right)
\end{aligned}
$$

Then the following statements are true.

a) If $(\eta, \vartheta) \in[0, \infty)^{2}$, then $I(\eta, \vartheta, 0)=0$, and if $\xi \neq 1$, then

$$
\begin{aligned}
I(1,1, \xi)= & (1+\xi) \ln \left(1+\xi^{2}\right)+(1-\xi) \ln (1-\xi)^{2} \\
& +2(1-\xi)\left(\arctan \frac{1+\xi}{1-\xi}-\frac{\pi}{4}\right) .
\end{aligned}
$$

b) If $(\eta, \vartheta, \xi) \in D_{I}$, then

$$
\begin{aligned}
\partial_{\xi} I(\eta, \vartheta, \xi)= & \vartheta \ln \left(\frac{(\eta+\xi \vartheta)^{2}+(1-\xi)^{2}}{(\eta-\xi \vartheta)^{2}+(1-\xi)^{2}}\right) \\
& -2\left(\arctan \frac{\eta+\xi \vartheta}{1-\xi}+\arctan \frac{\eta-\xi \vartheta}{1-\xi}-2 \arctan \eta\right)
\end{aligned}
$$

hence in particular $\partial_{\xi} I(\eta, \vartheta, 0)=0$ for $(\eta, \vartheta) \in[0, \infty)^{2}$.

c) If $(\eta, \vartheta, \xi) \in D_{I}$, then

$$
\partial_{\xi \xi}^{2} I(\eta, \vartheta, \xi)=-4 \eta \frac{\left(1-\xi\left(1+\vartheta^{2}\right)\right)^{2}+\eta^{2}-\left(1+\eta^{2}\right) \vartheta^{2}}{\left(\eta^{2}-\xi^{2} \vartheta^{2}\right)^{2}+(1-\xi)^{4}+2(1-\xi)^{2}\left(\eta^{2}+\xi^{2} \vartheta^{2}\right)}
$$

in particular

$$
\partial_{\xi \xi}^{2} I(\eta, \vartheta, 0)=-4 \eta\left(\frac{1-\vartheta^{2}}{1+\eta^{2}}\right)
$$


for $(\eta, \vartheta) \in[0, \infty)^{2}$, and

$$
\partial_{\xi \xi}^{2} I(1,1, \xi)=\frac{4 \xi}{(1-\xi)\left(1+\xi^{2}\right)}
$$

for $\xi \neq 1$.

d) If $(\eta, \vartheta, \xi) \in D_{I}$, then

$$
\begin{aligned}
\partial_{\xi \xi \xi}^{3} I(\eta, \vartheta, \xi)= & 8 \eta\left(\left(\eta^{2}-\xi^{2} \vartheta^{2}\right)^{2}+(1-\xi)^{4}+2(1-\xi)^{2}\left(\eta^{2}+\xi^{2} \vartheta^{2}\right)\right)^{-2} \\
& \times\left(\left(1-\xi\left(1+\vartheta^{2}\right)\right)\left((1-\xi)^{2}+\xi^{2} \vartheta^{2}\right)\right. \\
& \times\left(\left(\vartheta^{2}-1\right)\left((1-\xi)^{2}+\left(\eta^{2}-\xi^{2} \vartheta^{2}\right)\right)+4 \vartheta^{2} \xi(1-\xi)\right) \\
& +\left(1-\xi\left(1+\vartheta^{2}\right)\right)\left((1-\xi)^{2}+\left(\eta^{2}-\xi^{2} \vartheta^{2}\right)\right) \eta^{2}\left(1+\vartheta^{2}\right) \\
& +\left(\eta^{2}-\left(1+\eta^{2}\right) \vartheta^{2}\right) \\
& \times\left(-2\left(1-\xi\left(1-\vartheta^{2}\right)\right)\left((1-\xi)^{2}+\left(\eta^{2}-\xi^{2} \vartheta^{2}\right)\right)\right. \\
& \left.\left.-4(2 \xi-1) \vartheta^{2} \xi(1-\xi)\right)\right)
\end{aligned}
$$

e) If $(\eta, \vartheta) \in[0, \infty)^{2}$, and if $\xi \in[0,1)$, then by the Taylor formula

$$
I(\eta, \vartheta, \xi)=-2\left(1-\vartheta^{2}\right)\left(\frac{\eta}{1+\eta^{2}}\right) \xi^{2}+\frac{1}{2} \xi^{3} \int_{0}^{1}(1-s)^{2} \partial_{\xi \xi \xi}^{3} I(\eta, \vartheta, s \xi) d s
$$

Lemma A.3: The following statements are true.

a) If $(t, s, y, x) \in D_{k}$ with $t>0$, then

$$
s^{-1} k(t, s, y, x)=I(x / s, y / t, t / s)
$$

b) If $y, x \in C^{+}([0,1])$, then with $\tilde{y}(\tau)=\frac{y(\tau)}{\tau}$ and $\tilde{x}(\tau)=\frac{x(\tau)}{\tau}$ for $\tau \in(0,1]$, we have

$$
\int_{0}^{1} k(t, s, y(t), x(s)) d s=t^{2} \int_{t}^{\infty} \frac{d \xi}{\xi^{3}} I\left(\tilde{x}\left(\frac{t}{\xi}\right), \tilde{y}(t), \xi\right) \quad(t \in(0,1]) .
$$

c) For $(t, s, x) \in[0,1]^{3}$ with $s>0$ and $s \neq t$ we have $k(t, s, t, x) \geq 0$.

d) Let $h_{2}:[0, \infty) \backslash\{1\} \rightarrow[0, \infty)$ be defined by $h_{2}(\xi)=\xi+\left|\ln (1-\xi)^{2}\right|$. Then there is a constant $C>0$ such that $\left|\partial_{\eta} I(\eta, \vartheta, \xi)\right| \leq C h_{2}(\xi)$ for all $(\eta, \vartheta, \xi) \in[0,1]^{2} \times(1, \infty)$.

e) There is a function $h_{3}:[1, \infty) \rightarrow[0, \infty)$ such that $\left(\xi \mapsto \frac{h_{3}(\xi)}{\xi^{3}}\right) \in L_{1}([1, \infty))$ and $\left|\partial_{\vartheta} I(\eta, \vartheta, \xi)\right| \leq h_{3}(\xi)$ for $(\eta, \vartheta, \xi) \in[0,1]^{2} \times(1, \infty)$. 
f) There is a constant $C>0$ such that $\partial_{\xi \xi \xi}^{3} I(\eta, \vartheta, \xi) \mid \leq \frac{C}{(1-\xi)^{3}}$ holds for $(\eta, \vartheta) \in$ $[0,1]^{2}$ and $\xi \in[0,1)$.

g) There is a constant $C>0$ such that $\left|\partial_{\xi \xi \xi \eta}^{4} I(\eta, \vartheta, \xi)\right| \leq \frac{C}{(1-\xi)^{3}}$ holds for $(\eta, \vartheta) \in$ $\left[\frac{1}{2}, 1\right]^{2}$ and $\xi \in[0,1)$.

h) There is a constant $C>0$ such that $\left|\partial_{\xi \xi \xi \vartheta}^{4} I(\eta, \vartheta, \xi)\right| \leq \frac{C}{(1-\xi) 3}$ holds for $(\eta, \vartheta) \in$ $\left[\frac{1}{2}, 1\right]^{2}$ and $\xi \in[0,1)$.

i) There is a constant $C>0$ such that

$$
\frac{1}{4 \pi} \int_{1}^{\infty} \frac{d \xi}{\xi^{3}}\left|I\left(\tilde{x}\left(\frac{t}{\xi}\right), \tilde{y}(t), \xi\right)\right| \leq C
$$

and

$$
\frac{1}{8 \pi} \int_{t}^{1} d \xi \int_{0}^{1} d s(1-s)^{2}\left|\partial_{\xi \xi \xi}^{3} I\left(\tilde{x}\left(\frac{t}{\xi}\right), \tilde{y}(t), s \xi\right)\right| \leq C
$$

hold for all $t \in(0,1]$ and $x, y \in C^{+}([0,1])$ with $\tilde{x}(\tau)=\frac{x(\tau)}{\tau} \in[0,1]$ and $\tilde{y}(\tau)=\frac{y(\tau)}{\tau} \in$ $[0,1]$ for $\tau \in(0,1]$.
j) $\int_{1}^{\infty} \frac{d \xi}{\xi^{\xi}} I(1,1, \xi)=\pi+\ln 2$.
k) $\int_{0}^{1} d \xi \int_{0}^{1} d s(1-s)^{2} \partial_{\xi \xi \xi}^{3} I(1,1, s \xi)=\pi-2 \ln 2$.

Lemma A.4: Let $\Delta, \bar{\Delta} \in C^{+}([0,1])$ and $h(\xi)=\xi+\left|\ln (1-\xi)^{2}\right|$ for $\xi \in[0, \infty) \backslash\{1\}$. Then the following statements are true.
a) $\lim _{t \rightarrow 0^{+}}\left(\frac{1}{1-\ln (t)} \int_{t}^{1} \Delta(s) \frac{d s}{s}\right)=\Delta(0)$
b) $\sup _{t \in[0,1]}\left(\frac{1}{1-\ln (t)} \int_{t}^{1}|\Delta(s)-\bar{\Delta}(s)| \frac{d s}{s}\right) \leq|\Delta-\bar{\Delta}|$
c) $\lim _{t \rightarrow 0^{+}}\left(t^{-2} \int_{0}^{t} \Delta(s) h\left(\frac{t}{9}\right) s d s\right)=\left|\frac{h}{i d^{3}}\right|_{L_{1}((1, \infty))} \Delta(0)$
d) $\sup _{t \in[0,1]}\left(t^{-2} \int_{0}^{t}|\Delta(s)-\bar{\Delta}(s)| h\left(\frac{t}{s}\right) s d s\right) \leq\left|\frac{h}{i d^{s}}\right|_{L_{1}((1, \infty))}|\Delta-\bar{\Delta}|$
e) $\lim _{t \rightarrow 0^{+}}\left(-\int_{t}^{1} \frac{d s}{s} \ln (1-s) \Delta\left(\frac{t}{s}\right)\right)=\frac{\pi^{2}}{6} \Delta(0)$
f) $\sup _{t \in[0,1]}\left(-\int_{t}^{1} \frac{d s}{s} \ln (1-s)\left|\Delta\left(\frac{t}{s}\right)-\bar{\Delta}\left(\frac{t}{s}\right)\right|\right) \leq \frac{\pi^{2}}{6}|\Delta-\bar{\Delta}|$.

Acknowledgement: This paper is taken from our $\mathrm{Ph}$. D. thesis [4]. We wants to thank Prof. J. Batt, Prof. D. Lortz, and Dr. R. Kaiser for many interesting discussions. 


\section{References}

[1] Kaiser, R.: MHD equilibria with cusped plasma-vacuum interfaces (to appear).

[2] Kaiser, R. and D. Lortz: On the existence of plasma cormers. ZAMM 73 (1993), 165 171.

[3] Kaiser, R., Lortz, D. and G.O. Spies: Plasma corners. Part I. Phys. Fluids B4 (1992), $529-534$.

[4] Kunze, M.: Contributions to the Theory of Semicondensing Vector Fields in Banach Spaces with an Application to a Free Boundary Problem Arising in Plasma Physics. Ph. D. Thesis. München: Ludwig-Maximilian-Universität 1994.

[5] Kunze, M.: On zeros of semicondensing operators. Nonlin. Anal. (to appear).

[6] Lortz, D.: Plane free-boundary equilibria. Plasma Phys. Contr. Fusion 33 (1991), 77 - 89.

[7] Puel, J.P.: Sur un probleme de valeur propre non linéaire et de frontiere libre. C. R. Acad. Sci. Paris 484 (1977), $861 \cdot 863$.

[8] Spies, G.O.: Plasma comers. Part II. Phys. Fluids B4 (1992), 535 - 539.

[9] Temam, R.: Remarks on a free boundary value problem arising in plasma physics. Commun. Part. Diff. Equ. 2 (1977), 563 - 585.

Received 24.02.1994 